\title{
LACUNARY CONVERGENCE OF SERIES IN $L_{0}$ REVISITED
}

\author{
Lech DREWNOWSKI*
}

\begin{abstract}
A simpler proof is given for the recent result of I. Labuda and the author that a series in the space $L_{0}(\lambda)$ is subseries convergent if each of its lacunary subseries converges.
\end{abstract}

Let $(S, \Sigma, \lambda)$ be a measure space which we assume throughout to be locally finite (if $\lambda(A)>0$ then there is a $B \subset A$ with $0<\lambda(B)<\infty$ ) and such that the space $L_{0}(\lambda)$ is sequentially complete. Here $L_{0}(\lambda)$ denotes the topological vector space of all (equivalence classes) of scalar measurable functions on $S$ equipped with the topology of convergence in measure $\lambda$ on sets of finite measure. A subseries $\sum_{k} f_{n_{k}}$ of a given series $\sum_{n} f_{n}$ is said to be lacunary if $n_{k+1}-n_{k} \rightarrow \infty$. Recall that the subseries convergence and unconditional convergence of series coincide in sequentially complete topological vector spaces.

The purpose of this note is to present a simpler proof for the following result established in a recent joint paper of I. Labuda and the author [DL1]; see also [DL2] for its extension to the case of Bochner spaces $L_{0}(\lambda, E)$. The reader is referred to these two papers for historical information and relevant literature.

Theorem. The space $L_{0}(\lambda)$ has the Lacunary Convergence Property. That is, a series in $L_{0}(\lambda)$ is subseries convergent provided each of its lacunary subseries converges.

What we actually proved first was an analogue of the above result for positive series (see [DL2, Thm. 5.1]). That was relatively easy and

*The author was partially supported by the State Committee for Scientific Research (Poland), Grant no. 2 P03A 05115.

Key words and phrases: Space of all measurable functions, subseries convergence, lacunary subseries.

1991 Mathematics Subject Classification: 46E30, 40A30, 28A20.

Servicio de Publicaciones. Universidad Complutense. Madrid, 2000 
elementary, but our attempts to reduce somehow the case of general series to that of positive series failed. It took us quite a time before a proof for the general case was found, and it turned out to be quite technical and not so elementary. In particular, it crucially depended on a result of Orlicz asserting that

$$
\text { if a series } \begin{gathered}
\sum_{n} f_{n} \text { in } L_{0}(\lambda) \text { is unconditionally convergent, then } \\
\sum_{n}\left|f_{n}(s)\right|^{2}<\infty \text { a.e.; }
\end{gathered}
$$

see [O1, Hilfsatz] and, for stronger forms, [O2, Thm. 8], and [MO, Thm. 1]. (The proof in $[\mathrm{MO}]$ is particularly simple and self-contained.)

The proof presented here is also based on Orlicz's theorem, but formulated in a somewhat sharper form (stated below) which allows us for arguments very much like those used in the case of positive series. Roughly speaking, we work here with positive series of the form $\sum_{k}\left|\sum_{i \in N_{k}} f_{i}\right|^{2}$.

The sharper form of Orlicz's theorem needed here can easily be deduced from the former using the following general fact: If a series $\sum_{n} x_{n}$ in a topological vector space is subseries convergent, then for every sequence $\left(N_{k}\right)$ of disjoint subsets of $\mathbb{N}$ also the series $\sum_{k}\left(\sum_{i \in N_{k}} x_{i}\right)$ is subseries convergent.

Orlicz's Theorem. For a series $\sum_{n} f_{n}$ in $L_{0}(\lambda)$, the following are equivalent.

(a) $\sum_{n} f_{n}$ is subseries convergent.

(b) For every disjoint sequence $\left(N_{k}\right)$ of finite subsets of $\mathbb{N}$,

$$
\sum_{k=1}^{\infty}\left|\sum_{i \in N_{k}} f_{i}(s)\right|^{2}<\infty \quad \lambda \text {-a.e. }
$$

(c) For every disjoint sequence $\left(N_{k}\right)$ of finite subsets of $\mathbb{N}$,

$$
\sum_{i \in N_{k}} f_{i} \rightarrow 0 \quad \lambda \text {-a.e. }
$$


Let $K$ be a subset of $\mathbb{N}$. It is said to be $r$-rare for some $r \in \mathbb{N}$ if $\left|k-k^{\prime}\right| \geq r$ for all distinct $k, k^{\prime} \in K$; lacunary if for every $r \in \mathbb{N}$ there is $n$ such that the set $\{k \in K: k \geq n\}$ is $r$-rare. Evidently, if $K=\left\{n_{1}<n_{2}<\ldots\right\}$ is infinite, then it is lacunary iff $n_{k+1}-n_{k} \rightarrow \infty$. Also, as easily seen, $K$ is lacunary iff it is the union of a sequence $\left(K_{r}\right)$ of finite sets such that each $K_{r}$ is $r$-rare and $\max K_{r}+r \leq \min K_{r+1}$.

Proof of the Theorem. The basic idea of the proof is the same as in [DL1]: Assume that a series $\sum_{n} f_{n}$ in $L_{0}(\lambda)$ is not subseries convergent although all of its lacunary subseries converge. Then we construct a lacunary subseries $\sum_{k} f_{n_{k}}$ such that, for a suitable sequence $\left(g_{n}\right)$ of its "blocks", we have $\sum_{n}\left|g_{n}(s)\right|^{2}=\infty$ on a set of positive $\lambda$ measure. However, since also the series $\sum_{n} g_{n}$ is subseries convergent, this would contradict Orlicz's theorem. A more detailed argument runs as follows.

By Orlicz's Theorem, there is a disjoint sequence $\left(N_{k}\right)$ of finite subsets of $\mathbb{N}$ such that

$$
\sum_{k=1}^{\infty}\left|\sum_{i \in N_{k}} f_{i}(s)\right|^{2}=\infty \quad \text { on a set } D \text { with } 0<\lambda(D)<\infty .
$$

For $r=1,2, \ldots$ let

$$
A_{r j}=\{j+(k-1) r: k=1,2, \ldots\}, \quad j=1, \ldots, r .
$$

Obviously, for each $k$ the sets $M_{r j k}=A_{r j} \cap N_{k}(j=1, \ldots, r)$ form a partition of $N_{k}$. Furthermore, by the Cauchy-Schwartz inequality, we have

$$
\left(\sum_{k=1}^{\infty}\left|\sum_{i \in N_{k}} f_{i}(s)\right|^{2}\right)^{1 / 2} \leq \sum_{j=1}^{r}\left(\sum_{k=1}^{\infty}\left|\sum_{i \in M_{r j k}} f_{i}(s)\right|^{2}\right)^{1 / 2} .
$$

Therefore, if

$$
D_{r j}=\left\{s \in D: \sum_{k=1}^{\infty}\left|\sum_{i \in M_{r j k}} f_{i}(s)\right|^{2}=\infty\right\},
$$

then $D=D_{r 1} \cup \ldots \cup D_{r r}$. Before proceeding note the following easy consequence of Egoroff's theorem:

If a positive series $\sum_{k} h_{k}$ in $L_{0}(\lambda)$ is such that $\sum_{k} h_{k}(s)=\infty$ on a set $A$ of finite $\lambda$ measure, then for every $m \in \mathbb{N}$ and $\varepsilon>0$ there is an 
interval $I$ in $\mathbb{N}$ such that $m \leq \min I$ and $\sum_{k \in I} h_{k}(s) \geq 1$ on a measurable set $B \subset A$ with $\lambda(A \backslash B)<\varepsilon$.

Applying this inductively with respect to $r=1,2, \ldots$ and, for each $r$, with respect to $j=1, \ldots, r$ (taking $\varepsilon=2^{-r} / r$ and $E=D_{r j}$ ) to the series

$$
\sum_{k=1}^{\infty}\left|\sum_{i \in M_{r j k}} f_{i}(s)\right|^{2} \quad \text { on the set } D_{r j} \quad(j=1, \ldots, r),
$$

we find for each $r$ intervals $I_{r 1}, \ldots, I_{r r}$ in $\mathbb{N}$ and measurable sets $E_{r j} \subset$ $D_{r j}$ so that if

$$
\begin{gathered}
K_{r j}=\bigcup_{k \in I_{r j}} M_{r j k} \quad \text { for } j=1, \ldots, r, \\
K_{r}=K_{r 1} \cup \ldots \cup K_{r r}, \quad E_{r}=E_{r 1} \cup \ldots \cup E_{r r},
\end{gathered}
$$

then

$$
\begin{array}{ll}
\max K_{r j}+r<\min K_{r, j+1} & \text { for } j=1, \ldots, r-1, \\
\max K_{r}+r<\min K_{r+1}, & \lambda\left(D \backslash E_{r}\right)<2^{-r},
\end{array}
$$

and

$$
\sum_{k \in I_{r j}}\left|\sum_{i \in M_{r j k}} f_{i}(s)\right|^{2} \geq 1 \quad \text { on } E_{r j} \quad \text { for } j=1, \ldots, r .
$$

In consequence,

$$
\sum_{j=1}^{r} \sum_{k \in I_{r j}}\left|\sum_{i \in M_{r j k}} f_{i}(s)\right|^{2} \geq 1 \quad \text { on } E_{r} .
$$

Arrange all the sets $M_{r j k}$ in a single sequence $\left(M_{n}\right)$ so that for each $r$,

$$
\left\{M_{n}: p_{r} \leq n<p_{r+1}\right\}=\left\{M_{r j k}: j=1, \ldots, r, k \in I_{r j}\right\},
$$

where $1=p_{1}<p_{2}<\ldots ;$ note that $\left\{M_{n}: p_{r} \leq n<p_{r+1}\right\}$ is a partition of $K_{r}$. Then

$$
\sum_{p_{r} \leq n<p_{r+1}}\left|g_{n}(s)\right|^{2} \geq 1 \quad \text { on } E_{r}, \text { where } \quad g_{n}=\sum_{i \in M_{n}} f_{i}(s) .
$$


It follows that $\sum_{n=1}^{\infty}\left|g_{n}(s)\right|^{2}=\infty$ a.e. on $D$. Now, by the conditions above, the union $K$ of the sets $K_{r}$ is lacunary. Hence, by assumption, the series $\sum_{i \in K} f_{i}$ is subseries convergent, and so is the series $\sum_{n} g_{n}$. We have thus got a contradiction with Orlicz's theorem.

\section{References}

[DL1] L. Drewnowski and I. Labuda, Lacunary convergence of series in $L_{0}$, Proc. Amer. Math. Soc. 126 (1998), 1655-1659.

[DL2] - Vector series whose lacunary subseries converge, Studia Math. 138 (2000), 53-80.

[MO] W. Matuszewska and W. Orlicz, A note on modular spaces.IX, Bull. Acad. Polon. Sci., Sér. Sci. Math. Astronom. Phys. 16 (1968), 801-808.

[O1] W. Orlicz, Über die Divergenz von allgemeinen Orthogonalreihen, Studia Math. 4 (1933), 27-32.

[O2] On a class of asymptotically divergent sequences of functions, Studia Math. 12 (1951), 286-307.

Faculty of Mathematics and Computer Science

A. Mickiewicz University

Matejki 48/49, 60-769 Poznań

Poland

E-mail address: drewlech@amu.edu.pI

Recibido: 5 de Marzo de 1999 